\title{
Structural changes in white matter lesion patients and their correlation with cognitive impairment
}

This article was published in the following Dove Medical Press journal:

Neuropsychiatric Disease and Treatment

\author{
Jinfang Wang ${ }^{1,2}$ \\ Yi Liang ${ }^{2}$ \\ Hongyan Chen' \\ Wanming Wang ${ }^{2}$ \\ Yanwen Wang ${ }^{2}$ \\ Ying Liang ${ }^{3}$ \\ Yumei Zhang ${ }^{1,4}$
}

'Department of Neurology, Beijing Tiantan Hospital, Capital Medical University, Beijing I00050, China; 2Department of Neurology, General Hospital of The Yang Tze River Shipping, Wuhan Brain Hospital, Wuhan 430000, China; ${ }^{3}$ School of Biomedical Engineering, Capital Medical University, Beijing 100050 , China; ${ }^{4}$ Department of Rehabilitation Medicine, Beijing Tiantan Hospital, Capital Medical University, Beijing I00050, China
Correspondence: Yumei Zhang Department of Rehabilitation Medicine, Beijing Tiantan Hospital, Capital Medical University, Beijing, 10050, China

Tel +13691404106

Email zhangyumei95@aliyun.com
Background: White matter lesions (WMLs) play a role in cognitive decline and dementia. Little is known about gray matter (GM) changes in WMLs. This study aimed to investigate GM changes in WML patients.

Materials and methods: Correlations between altered structural volume and cognitive assessment scores were investigated. GM and white matter (WM) changes in 23 WML-vascular dementia (VaD) patients, 22 WML-non-dementia vascular cognitive impairment (VCIND) patients, and 23 healthy control (HC) subjects were examined. Gray matter density (GMD) was calculated by measuring local proportions of GM at thousands of homologous cortical locations. WM volume was obtained by fully automated software using voxel-based morphometry (VBM). Results: Widespread GMD was significantly lower in WML patients compared to control subjects in cortical and subcortical regions $(p<0.05)$. Greatest differences were found in the bilateral anterior cingulate cortex, inferior frontal gyrus, insula, angular gyrus, caudate, precentral gyrus, and right middle temporal gyrus, right thalamus. Secondary region of interest (ROI) analysis indicated significantly greater GMD in the bilateral caudate among WML-VCIND patients $(\mathrm{n}=22)$ compared to HCs $(p<0.05)$. There was a significant difference in WM volume between WML patients and control subjects $(p<0.05)$. Greatest differences were located in the genu/body/splenium of the corpus callosum and superior corona radiata L, and posterior corona radiata $\mathrm{L}$. There was a significant association between structural changes and cognitive scores (Montreal Cognitive Assessment [MoCA] score) $(p<0.05)$. There was no significant correlation between structural changes and Mini Mental State Examination (MMSE) scores $(p>0.05)$. Conclusion: GMD and WM volume were changed in WMLs, and the changes were detectable. Correlation between structural changes and cognitive function was promising in understanding the pathological and physiological mechanisms of WMLs.

Keywords: white matter lesions, vascular dementia, gray matter density, dementia

\section{Introduction}

White matter lesions (WMLs), which can be studied in vivo by imaging techniques, are related to cognitive impairment. ${ }^{1,2}$ Although other studies observed that severe WML is not necessarily associated with cognitive impairment, ${ }^{3,4}$ some studies suggest that clinically, WMLs act as risk factors for cortical and the subcortical brain atrophy. 5,6 Neuronal activity is related to gray matter (GM) activity. The complex structural abnormalities of GM in WML patients may be the explanation for the association between WML and cognitive impairment.

Structural MRI can provide morphological information that reveals the cortical and subcortical deformation in response to pathological attacks. Voxel-based morphometry (VBM) is an automated technique that offers rapid unbiased assessment of local brain tissue changes on a whole-brain level. ${ }^{7,8}$ VBM has recently been successfully used 
to characterize the structural brain differences in several degenerative brain disorders. ${ }^{9-11}$

With respect to cognitive impairment, Seo et $\mathrm{al}^{12}$ found that the subcortical vascular mild cognitive impairment patients showed cortical thinning in inferior frontal. Li et al ${ }^{13}$ also found that subcortical vascular mild cognitive impairment exhibited widespread abnormalities in structural volume. WML is one of the most common causes of subcortical vascular mild cognitive impairment. ${ }^{14}$ Therefore, identifying the GM changes over the whole brain in WML patients is very important.

Therefore, our study was intended to determine whether structural abnormalities exist in the gray matter density (GMD) and white matter (WM) volume of WML patients using the VBM technique. We hypothesized that there would be alterations in GMD in WML patients compared with healthy controls (HCs). Furthermore, we examined whether correlations existed between structural changes and cognitive assessment scores.

\section{Materials and methods Subjects}

Forty-five patients with WMLs were recruited from Beijing Tiantan Hospital, Capital Medical University, China. The diagnosis of WMLs was made unanimously by two radiologists who independently evaluated the fluid-attenuated inversion recovery (FLAIR) MR images visually without the knowledge of the participants' clinical profiles. Twenty-three HCs were recruited with age, gender, and education levels matching the patients. The inclusion criteria for the WML patients were as follows: 1) patients aged between 50 and 85 years, 2) patients who showed WMLs on MRI scans according to a revised version of the scale of Fazekas, and 3) presence of a contactable informant throughout the study. ${ }^{15}$ The exclusion criteria for the WML patients were as follows (WMLs with the following comorbidities should be excluded): 1) cardiac or renal failure, cancer, or other severe systemic diseases, 2) unrelated neurological diseases such as epilepsy, traumatic brain injury, and multiple sclerosis, 3) chronic cerebral infarction or other lesions, 4) leukoencephalopathy of non-vascular origin, 5) dementia of nonvascular origin, 6) psychiatric diseases or drug addiction, 7) consciousness disruption or aphasia, or 8 ) inability or refusal to undergo brain MRI.

This study was approved by the Ethics Committee of Beijing Tiantan Hospital, Capital Medical University, Beijing, China. All the patients have written the consent forms and approved this study. This study was conducted in accordance with the Declaration of Helsinki.

\section{Clinical cognitive assessment}

All subjects were instructed to complete the Chinese version of Mini Mental State Examination (MMSE), the Beijing version of Montreal Cognitive Assessment (MoCA), and the Clinical Dementia Rating scale (CDR) under the supervision of a physician. ${ }^{16,17}$ The tests were completed in a strict order in accordance with the standard protocols in a quiet room. The following education-specific reference cutoff values for MMSE scores were used: middle and high-27, elementary-24, and illiterate-21. ${ }^{18}$ For MoCA, the cutoff value for cognitive impairment was less than $26 .{ }^{19}$ In addition, one additional point was added to the raw MoCA score when the subject's education years were less than $12 .{ }^{18}$

Based on the results of these cognitive tests, the subjects were divided into three groups, including WML patients with non-dementia vascular cognitive impairment (WMLVCIND, $n=22$ ), WML patients with vascular dementia (WML-VaD, $\mathrm{n}=23$ ), and $\mathrm{HC}$ subjects with normal cognition (HC, $\mathrm{n}=23$ ). The WML-VCIND patients complied with the following criteria: 1) $\mathrm{CDR}=0.5$, 2) $24 \leq \mathrm{MMSE}<27$ with years of education $\geq 6$, or $20 \leq$ MMSE $<24$ with years of education $<6$, or $17 \leq$ MMSE $<21$ with years of education $=0$, and 3) $\mathrm{MoCA}<26$. The WML-VaD patients complied with the following criteria: 1) $\mathrm{CDR} \geq 1,2$ ) MMSE $<24$ with years of education $\geq 6$, or MMSE $<20$ with years of education $<6$, or MMSE $<17$ with years of education $=0$, and 3) MoCA $<22$. The HCs complied with the following criteria: 1) MRI showed normal brain structure, 2) $\mathrm{CDR}=0,3$ ) $\mathrm{MMSE} \geq 27$ with years of education $\geq 6$, or MMSE $\geq 24$ with years of education $<6$, or MMSE $\geq 21$ with years of education $=0$, and 4) $\mathrm{MoCA} \geq 26$.

\section{Acquisition of images}

All the subjects were analyzed and scanned by utilizing a 3 Tesla Siemens Magnetom Verio MRI scanner. A T2WFLAIR sequence was applied to detect WMLs. A standard T1-weighted 3D magnetization prepared rapid gradient echo sequence was applied with repetition time (TR) $=2,300 \mathrm{~ms}$, echo time $(\mathrm{TE})=3.28 \mathrm{~ms}$, time inversion $(\mathrm{TI})=1,200 \mathrm{~ms}$, matrix size $=256 \times 256$, flip angle $(\mathrm{FA})=9^{\circ}$, slice thickness $=1 \mathrm{~mm}$, interslice gap $=0.5 \mathrm{~mm}$, and number of slices $=256$. Rubber earplugs were used to reduce noise, and foam cushions were used to fix their head to minimize potential motion artifacts.

\section{Processing of images}

We used the VBM8 toolbox of the Statistical Parametric Mapping (SPM) software (http://www.fil.ion.ucl.ac.uk/spm/) to analyze the T1 data. $\mathrm{T} 1$ images were first segmented into 
the WM, GM, and cerebrospinal fluid (CSF) by utilizing the unified segmentation module. These segmented GM and WM images were then used to obtain a more accurate intersubject registration model using DARTEL. This model alternates between computing a group template and warping an individual's tissue probability maps into alignment with this template and ultimately creates an individual flow field of each participant. Then, each participant's images were normalized into the Montreal Neurological Institute space with the normalized images modulated to ensure that relative GM and WM volumes were well preserved following spatial normalization. Finally, these images were smoothed with an $8 \mathrm{~mm}$ full width at half maximum Gaussian kernel.

\section{Statistical analysis}

Statistical analysis was performed in SPSS Statistics V.19.0 (IBM Corporation, Armonk, NY, USA). Demographic and behavioral differences between groups were tested by ANOVA. In VBM analysis, ANOVA was used to calculate the voxel-wise GM and WM volume differences among WML-vascular dementia (VaD), WML-non-dementia vascular cognitive impairment (VCIND), and normal control groups (NC). The significance threshold was set at $p<0.05$ (family-wise error [FWE] corrected for multiple comparisons performed by permutation test with threshold-free cluster enhancement [TFCE]). Then, post hoc Tukey-Kramer/HSD test was carried out to further explore the possible betweengroup differences in regions with significant ANOVA results. The statistical significance for post hoc test was set at $p<0.001$ and cluster size $>50$. Then, the difference in mean values in WMLs and controls was tested by ANOVA.
Significant clusters obtained from both the WM and the GM were analyzed to evaluate the differences among the WML$\mathrm{VaD}$, WML-VCIND, and HC groups. Then, comparisons of significant clusters of the region of interest (ROI) were carried out. In order to clarify the correlations among all the predictor variable differences, GMD, WM volumes, and the cognitions, the multiple linear regression analysis was employed to evaluate the critical predictor variables, white and GM clusters, age, sex, and the levels of education. First, each outcome variable of education, hypertension, and age were regressed in this study. The relationship between WM volume and GMD was also tested.

\section{Results}

\section{Demographic and clinical data}

The results showed that there were no significant differences between WML and HC groups for the demographic data $(p=0.001)$, but not for the age (Table 1). The mean age of WMLs was older compared to that in HC groups. The WML patients showed significantly lower scores for the MMSE $(p=0.000)$ and MoCA $(p=0.000)$.

\section{Changes in GMD in cortical and subcortical GM}

VBM illustrated extensive and significant differences of the GMD in WML patients compared to that of the HC group $(p<0.001)$ (Figure 1A and Table 2), which included one cluster of high density in the WML group. The WML-VaD patients exhibited decreased GMD in cortical regions (Figure 1B), which included the frontal gyrus, cingulate cortex, and temporal and occipital gyrus. Compared to WML-mild cognitive impairment, the differences were also in the cortical regions

Table I Demographic data of the WML-VCIND, WML-VaD, and healthy control subjects

\begin{tabular}{|c|c|c|c|c|c|}
\hline Variables & $N C(n=23)$ & WML-VCIND $(n=22)$ & WML-VaD (n=23) & Statistics & $P$-value \\
\hline Age, years & $55.34 \pm 10.07$ & $63.14 \pm 7.36$ & $65.35 \pm 8.82$ & 8.09 & 0.001 \\
\hline Women, n (\%) & II (47.83) & $10(45.45)$ & $7(30.43)$ & 1.682 & 0.431 \\
\hline \multicolumn{6}{|l|}{ Education, n (\%) } \\
\hline Primary & 0 & I (4.54) & $3(13.04)$ & 1.682 & 0.431 \\
\hline Secondary & $12(52.17)$ & $14(63.64)$ & $16(69.56)$ & & \\
\hline Superior & II (47.83) & $7(31.82)$ & $4(17.40)$ & & \\
\hline Hypertension, n (\%) & $9(39.13)$ & $6(27.27)$ & $14(60.87)$ & 5.265 & 0.068 \\
\hline Smoking, n (\%) & 6 & 5 & 4 & 0.514 & 0.773 \\
\hline Diabetes, n (\%) & 4 & 2 & 5 & 1.364 & 0.505 \\
\hline Cholesterol, n (\%) & 5 & 3 & 6 & 1.094 & 0.579 \\
\hline Body mass index & $23.87 \pm 1.18$ & $24.27 \pm 1.12$ & $24.13 \pm 1.01$ & 0.772 & 0.466 \\
\hline MMSE & $28.9 I \pm 1.31$ & $25.54 \pm 1.84$ & $22.04 \pm 1.99$ & 89.89 & 0.000 \\
\hline MoCA & $27.65 \pm 1.49$ & $22.23 \pm 1.45$ & $17.39 \pm 2.92$ & 140.407 & 0.000 \\
\hline
\end{tabular}

Note: Numbers represent mean \pm SD.

Abbreviations: MMSE, Mini Mental State Examination; MoCA, Montreal Cognitive Assessment; WML, white matter lesion; VaD, vascular dementia; VCIND, non-dementia vascular cognitive impairment. 
A

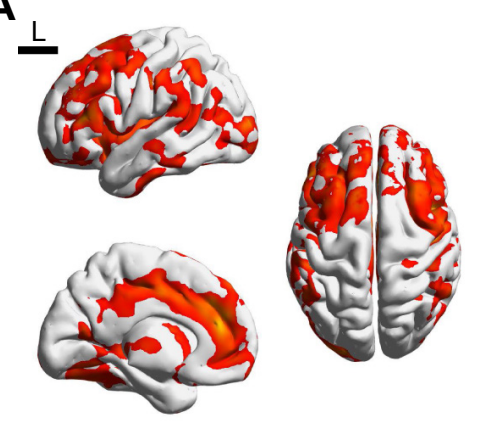

B

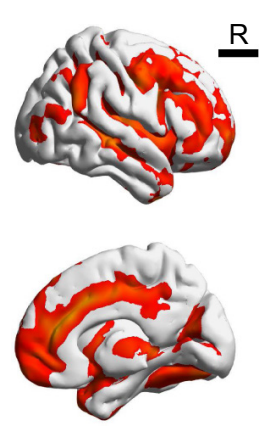

23.67

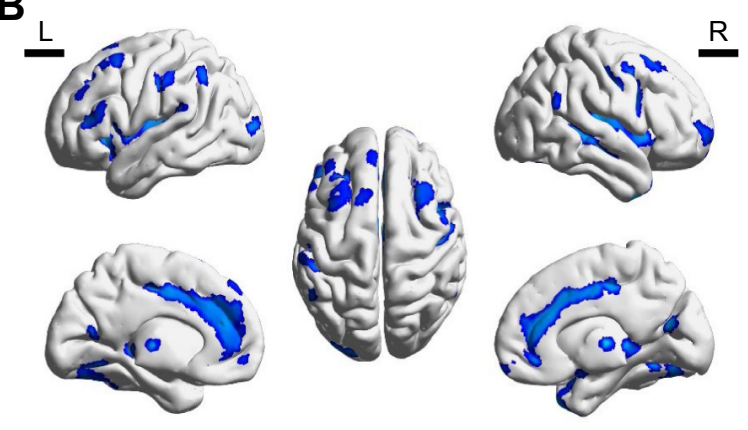

3.82

\section{(1)}

C
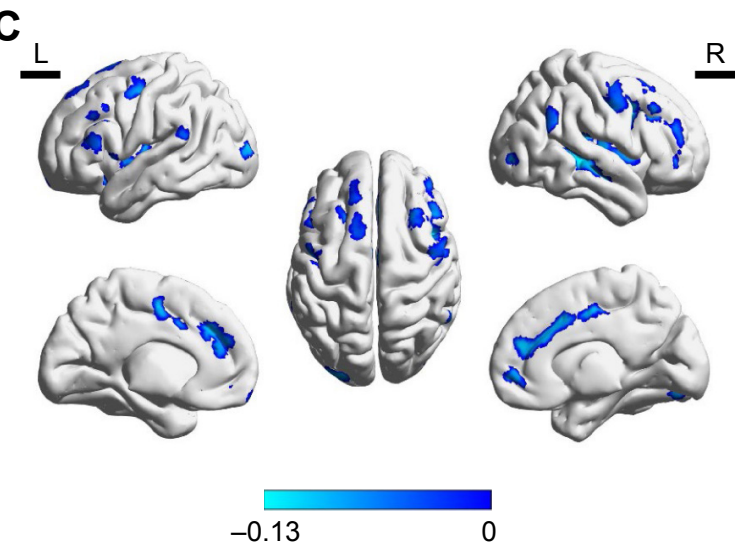

0

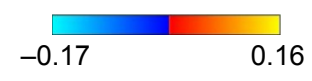

D
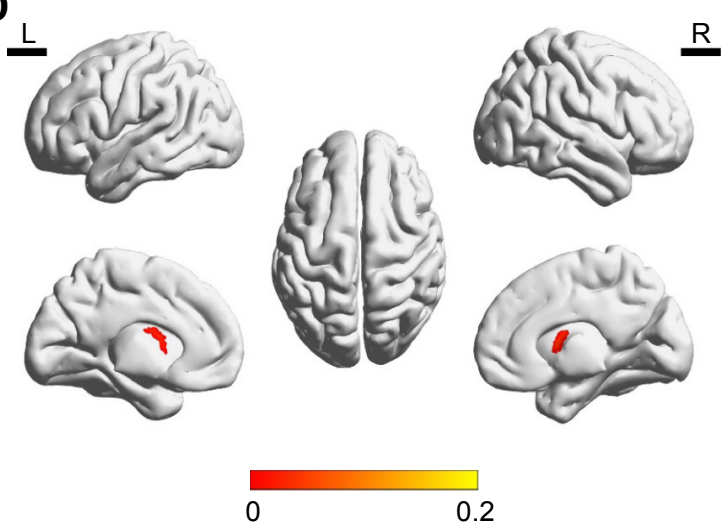

Figure I Differences in GMD.

Notes: (A) GMD in WML patients. (B) GMD in cortical regions of WML-VaD patients. (C) GMD in cortical regions of WML-MCl. (D) GMD in caudate of WML-MCI patients.

Abbreviations: GM, gray matter; GMD, gray matter density; WML, white matter lesion; $\mathrm{VaD}$, vascular dementia; $\mathrm{MCl}$, mild cognitive improvement.

Table 2 Regions showing GMD changes in WML patients

\begin{tabular}{|l|l|l|l|}
\hline \multirow{2}{*}{ Brain regions } & \multicolumn{3}{|l|}{ MNI coordinates $(\mathbf{m m})$} \\
\cline { 2 - 4 } & $\boldsymbol{x}$ & $\boldsymbol{y}$ & $\mathbf{z}$ \\
\hline ACC.L & -3 & $4 \mathrm{I}$ & 20 \\
\hline ACC.R & 6 & 37 & 20 \\
\hline IFG.L & -49 & $3 \mathrm{I}$ & 13 \\
\hline IFG.R & 46 & 42 & 15 \\
\hline INS.L & -42 & 6 & 0 \\
\hline INS.R & 44 & 7 & -2 \\
\hline MTG.R & 58 & -27 & -5 \\
\hline ANG.L & -52 & $-5 I$ & 48 \\
\hline ANG.R & 58 & $-5 I$ & 31 \\
\hline CAU.L & -11 & 8 & 12 \\
\hline CAU.R & 13 & 6 & 12 \\
\hline THA.R & 6 & -20 & 4 \\
\hline PrCG.L & -47 & -1 & 50 \\
\hline PrCG.R & 53 & 0 & 48 \\
\hline
\end{tabular}

Abbreviations: ACC, anterior cingulate cortex; ANG, angular gyrus; CAU, caudate; GMD, gray matter density; IFG, inferior frontal gyrus; INS, insula; MTG, middle temporal gyrus; PrCG, precentral gyrus; THA, thalamus; WML, white matter lesion.
(Figure 1C). The subcortical region is mainly located in the caudate. Interestingly, the WML-MCI patients showed increased GMD in the caudate compared to the HCs (Figure 1D).

\section{Changes in WM volumes}

We found differences in clusters in the WM analysis (Table 3 and Figure 2). Particularly, the WML-VaD patients exhibited a significant lower volume in four clusters (Table 4

Table 3 Different regions in white matter volume between WMLs and HC: coordinates are defined in standard space

\begin{tabular}{|l|l|l|l|}
\hline Brain regions & Volume $\left(\mathbf{m m}^{3}\right)$ & $\boldsymbol{F}$ & $\begin{array}{l}\text { MNI coordinates } \\
(\mathbf{m m})\end{array}$ \\
\hline sCC & 768 & 13.314 & 3306 \\
\hline gCC & $1,52 \mathrm{I}$ & 10.445 & $5-39$ I7 \\
\hline ACR.L & 585 & 9.892 & $-26-5327$ \\
\hline PCR.L & $24 \mathrm{I}$ & $7.94 \mathrm{I}$ & $-27-1 \mathrm{I} 39$ \\
\hline
\end{tabular}

Abbreviations: $\mathrm{HC}$, healthy control; WML, white matter lesion; sCC, splenium of corpus callosum; gCC, genu of corpus callosum; ACR.L, left anterior corona radiata; PCR.L, left posterior corona radiata. 
WMLs
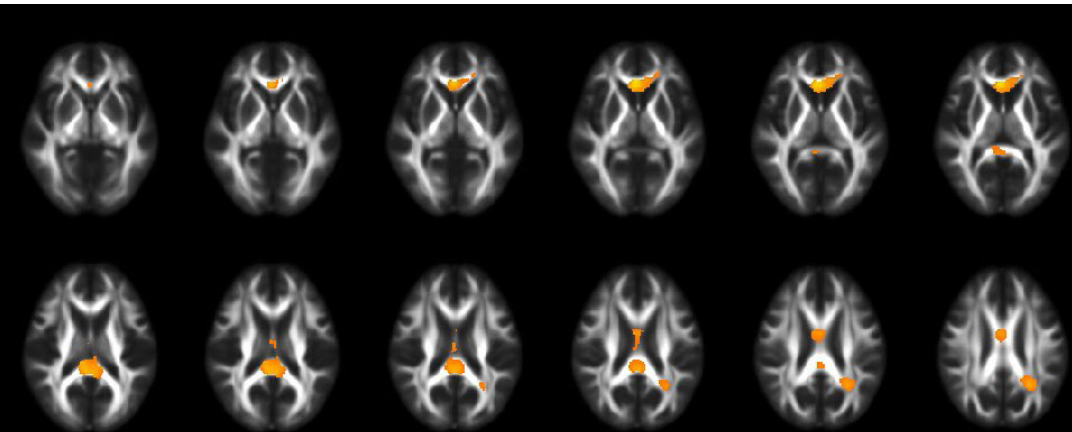

HC

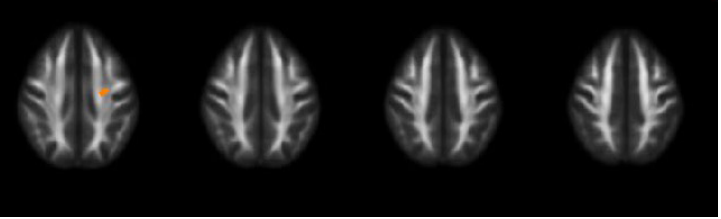

Figure 2 White matter volume difference between WMLs and HC. Abbreviations: $\mathrm{HC}$, healthy control; WML, white matter lesion.

and Figure 3). The abovementioned four clusters mainly comprised the following fibers: 1) genu/body/splenium of corpus callosum $(F=10.045, p=0.000)$ and 2$)$ superior corona radiata $\mathrm{L}$, posterior corona radiata $\mathrm{L}(F=9.871, p=0.000)$.

\section{Correlation of gray and WM changes with cognitive impairment}

Higher white matter density (WMD) was associated with lower GMD, most prominently in the ACC.L and CAU.R (Figure 4). As shown in Table 5, $\mathrm{sCC}(r=0.344, p=0.004)$, $\operatorname{gCC}(r=0.284, p=0.02)$, ACR.L $(r=0.34, p=0.005)$, and PCR.L $(r=0.401, p=0.001)$ were significantly correlated with the MoCA score $(p<0.05)$. But, there were no global metrics with significant correlation with MMSE (Table 5).

\section{Discussion}

To our knowledge, our study is the first study to combine the GM and WM volume abnormalities in patients with WMLs.
The main findings were listed as follows: 1) Compared with HCs, patients with WMLs showed decreased GMD in the frontal gyrus, cingulate cortex, temporal and occipital gyrus, and the subcortical region mainly located in the caudate. 2) Compared with $\mathrm{HCs}$, patients with WMLs showed a significantly lower WM volume in the genu/body/splenium of the corpus callosum, superior corona radiata L, and posterior corona radiata L. 3) The higher WMG was related to the lower density of GM. Summarily, the abovementioned results hint that WMLs could predict the brain structure changes associated with the cognitive function, including caudate and frontal lobes.

WMLs are prevalent in older population, particularly in populations aged 60 years. ${ }^{20}$ In this study, we also found that the mean age of the WML group was more than 60 years. Aging populations tend to have higher WML burden, patients with cognitive impairment often have an even higher WML burden. ${ }^{21}$ Multiple cross-sectional studies have documented

Table 4 WM volume difference between WMLs and NC

\begin{tabular}{|l|l|l|l|l|l|}
\hline \multirow{2}{*}{ WM region } & \multicolumn{2}{|l|}{ Mean \pm SD } & \multirow{2}{*}{$\boldsymbol{F}_{2,65}$} & \multirow{2}{*}{-value } \\
\cline { 2 - 5 } & WML-VaD & WML-VCIND & NC & & \\
\hline $\mathrm{sCC}$ & $0.527 \pm 0.072$ & $0.644 \pm 0.101$ & $0.583 \pm 0.088$ & 10.045 & 0.000 \\
\hline $\mathrm{gCC}$ & $0.451 \pm 0.072$ & $0.543 \pm 0.077$ & $0.521 \pm 0.069$ & 9.871 & 0.000 \\
\hline ACR.L & $0.621 \pm 0.166$ & $0.763 \pm 0.119$ & $0.756 \pm 0.095$ & 8.697 & 0.000 \\
\hline PCR.L & $0.671 \pm 0.122$ & $0.78 \pm 0.084$ & $0.779 \pm 0.109$ & 7.856 & 0.001 \\
\hline
\end{tabular}

Abbreviations: NC, normal control; WM, white matter; WML, white matter lesion; VaD, vascular dementia; VCIND, non-dementia vascular cognitive impairment. 

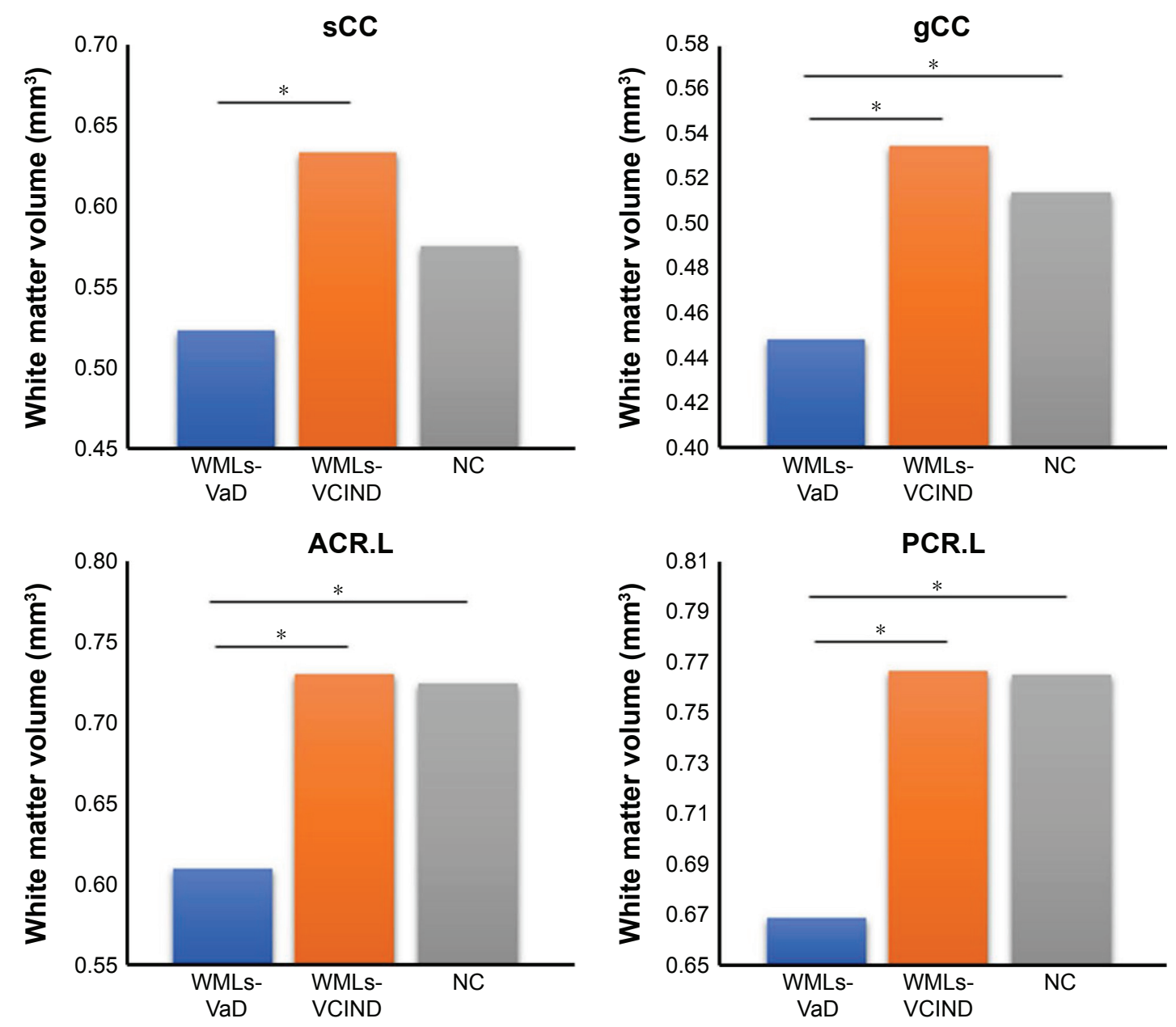

Figure 3 Histogram of white matter volume differences between WMLs and HC.

Note: $* p<0.05$.

Abbreviations: sCC, splenium of corpus callosum; gCC, genu of corpus callosum; ACR.L, left anterior corona radiata; PCR.L, left posterior corona radiata; WMLs-VCIND, WMLs patients with vascular no dementia; WMLs-VaD, WMLs patients with vascular dementia; NC, normal control; WML, white matter lesion.

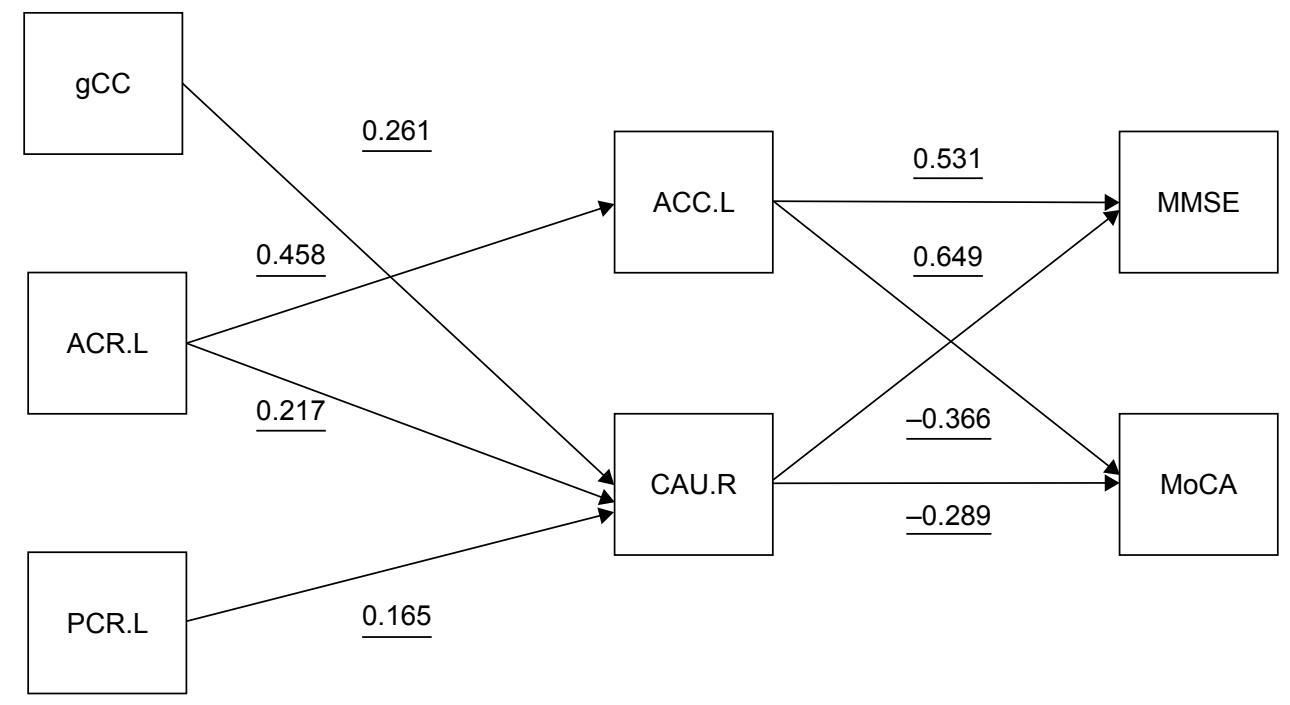

Figure 4 Regression-based model of relationships among gray matter density, white matter volume, and MMSE/MoCA scores.

Notes: Gray matter density was extracted using the VBM in SPM5. The predictor variables derived from multiple linear regression $(p<0.05)$. The direction of the arrows is based on the relationship between pairs of variables.

Abbreviations: MMSE, Mini Mental State Examination; MoCA, Montreal Cognitive Assessment; SPM, Statistical Parametric Mapping; VBM, voxel-based morphometry; ACC.L, left anterior cingulate cortex; ACC.R, right anterior cingulate cortex; ACR.L, left anterior corona radiata; PCR.L, left posterior corona radiata. 
Table 5 Correlation between $\mathrm{WH}$ changes and the cognitive impairment

\begin{tabular}{|l|l|l|l|l|}
\hline \multirow{2}{*}{$\begin{array}{l}\text { WM } \\
\text { regions }\end{array}$} & \multicolumn{3}{|l|}{ MoCA } & \multicolumn{2}{l|}{ MMSE } \\
\cline { 2 - 5 } & $\boldsymbol{r}$ & P-value & $\boldsymbol{r}$ & P-value \\
\hline sCC & 0.344 & 0.004 & - & $>0.05$ \\
\hline gCC & 0.284 & 0.02 & - & $>0.05$ \\
\hline ACR.L & 0.34 & 0.005 & - & $>0.05$ \\
\hline PCR.L & 0.401 & 0.001 & - & $>0.05$ \\
\hline
\end{tabular}

Note: The MoCA and MMSE were evaluated at the different periods.

Abbreviations: sCC, splenium of corpus callosum; gCC, genu of corpus callosum; ACR.L, left anterior corona radiata; PCR.L, left posterior corona radiata; MMSE, Mini Mental State Examination; MoCA, Montreal Cognitive Assessment; WM, white matter.

the relationships between the cognition and the WMLs in the elderly individuals with normal cognitions. ${ }^{22-25}$ Previous study has found that higher WMLs reflecting greater cerebral small vessel disease are correlated with the lower volume of GM. ${ }^{26}$ Meanwhile, the WMLs were also associated inversely with the GM volume in cognitively normal elders. ${ }^{27}$ The GM atrophy is correlated with the higher WML in patients. ${ }^{28}$ A previous study also showed that in WMLs there was significant correlation between executive dysfunction and GM atrophy. ${ }^{28}$ Our study also demonstrated that WMLs affect the GMD, which is associated with the cognitive assessment scores. Our study advanced the relationship of WMLs with specific regional damage. The higher WMG always triggers greater loss of the GM volume and lower neuropsychological test scores. These findings contributed to our understanding of the relationships among WMLs, GM atrophy, and cognitive impairment in patients.

Consistent with previous studies, our studies showed that WMLs were associated with the decreased volume of frontal lobe regions and cognitive decline..$^{5,28-32}$ Our results indicated that there was a significant association between structural changes and cognitive scores (MoCA score). However, there were no significant correlations between structural changes splenium of corpus callosum (SCC), genu of corpus callosum ( $\mathrm{gCC}$ ), left anterior corona radiata (ACR.L) and left posterior corona radiata (PCR.L) and MMSE scores in the present WML patients. It is well known that the frontal lobe circuit including the frontal lobe is a core neural pathway representing the pathophysiological feature of cognition. ${ }^{28}$ Particularly, the associated fibers within subcortical pathways and frontal lobe have been associated with executive functions. ${ }^{33,34}$ There are also a few tract connect areas in occipital cortex, frontal lobe, and temporal cortex. Meanwhile, the fibers play critical roles in the processing and integrating of the information, being the core feature of the cognitive dysfunction. ${ }^{35} \mathrm{In}$ agreement with these findings, patients with WMLs showed decreased GMD in frontal gyrus, cingulate cortex, and temporal and occipital gyrus in this study. In this sense, the WM tracts interconnect different lobes, suggesting that brain is a broad neural circuit. In this condition, the WMLs might be considered to be the imaging indicators of the potential or the underlying GM atrophy. GM tissue loss might subsequently cause the lower cognitive reserve. ${ }^{36,37}$ Figure 4 presents the possible pathway to cognitive impairment, the GM atrophy, or the disconnection of the neural circuit. Understanding these processes will contribute to our insight on preventing cognitive impairment. However, there are no reports of structural alterations in subcortical regions of WML patients to date. In our study, we found that the subcortical caudate exhibited increased GM volume compared to HCs, providing complementary evidence to the cognition dysfunction pathway of the aberrant fronto-subcortical circuits.

It is critical that the differences for the volume of WM between WML-MCI and WML-VaD were significant. As we expected, patients with different levels of cognition would differ from each other. However, these changes provided a promising biomarker for the two different illness trajectories.

Moreover, WMLs are often found in the other neurological disorders or cardiovascular diseases, such as migraine and multiple sclerosis. ${ }^{38}$ The neurological or cardiovascular disorders might be associated with the WMLs in the patients. In the investigations that follow, we would investigate the correlations between the other WMLs occurred patients and the cognitive impairment.

There are several limitations in this study. First, the current study was cross-sectional. Second, we analyzed the whole brain WM volume, which cannot rate the extent of WMLs. In this study, we attempted to collect plenty of clinical information and data so as to confirm the illness trajectories. Our findings are consistent with those of the previous studies. Third, the detailed patient comorbidities have not been illustrated, and their correlations with cognitive impairment have been investigated. In the study that follows, we would profile a correlation between patient comorbidities, WMLs, and cognitive impairment. Fourth, the T1 data were analyzed by using the VBM8 toolbox of the SPM software, which might misinterpret the WMLs as the GM, especially when they are hypointense in T1 sequences. We would employ the Lesion Segmentation Tool (LST) to analyze the T1 data in the future study.

\section{Conclusion}

The present study provided a few evidence that WMLs is related to GM alterations and cognition. These changes affect 
both cortical and subcortical regions, leading to the aberrant neural cognition pathways. Regardless, these results provided empirical evidence to support neural correlates of WMLs and cognition dysfunction.

\section{Acknowledgments}

This study was granted by the Beijing Municipal Administration of Hospitals Clinical Medicine Development of Special Funding Support (ZYLX201836), National Key Technology Research and Development Program of China ( 2018YFC2002300, 2018YFC 2002302, 2015BAI12B02), the National Natural Science Foundation of China (NSFC: 81371201, 31600933) National Key Technology Research and Development Program of the Ministry of Science and Technology of The People's Republic of China (2015BAI12B04) and Hubei Province health and family planning scientific research project (Grant No. WJ2017M208,WJ2017F057).

\section{Disclosure}

The authors report no conflicts of interest in this work.

\section{References}

1. Guo Y, Wang S, Jiang B, et al. Encephalitis with reversible splenial and deep cerebral white matter lesions associated with Epstein-Barr virus infection in adults. Neuropsychiatr Dis Treat. 2017;13(1):2085-2092. doi:10.2147/NDT.S135510

2. The LADIS Study Group; Poggesi A, Pantoni L, Inzitari D, et al. 2001-2011: a decade of the LADIS (Leukoaraiosis And DISability) Study. What have we learned about white matter changes and smallvessel disease? Cerebrovasc Dis. 2011;32(6):577-588. doi:10.1159/ 000334498

3. Sabri O, Ringelstein EB, Hellwig D, et al. Neuropsychological impairment correlates with hypoperfusion and hypometabolism but not with severity of white matter lesions on MRI in patients with cerebral microangiopathy. Stroke. 1999;30(3):556-566.

4. Fein G, Van DC, Davenport L, et al. Preservation of normal cognitive functioning in elderly subjects with extensive white-matter lesions of long duration. Arch Gen Psychiatry. 1990;47(3):220-223.

5. Appelman AP, Exalto LG, van der Graaf Y, Biessels GJ, Mali WP, Geerlings MI. White matter lesions and brain atrophy: more than shared risk factors? A systematic review. Cerebrovasc Dis. 2009;28(3): 227-242. doi:10.1159/000226774

6. Mok V, Wong KK, Xiong Y, et al. Cortical and frontal atrophy are associated with cognitive impairment in age-related confluent whitematter lesion. J Neurol Neurosurg Psychiatry. 2011;82(1):52-57. doi:10.1136/jnnp.2009.201665

7. Ashburner J, Friston KJ. Voxel-based morphometry-the methods. Neuroimage. 2000;11(6 Pt 1):805-821. doi:10.1006/nimg.2000. 0582

8. Ashburner J, Friston KJ. Why voxel-based morphometry should be used. Neuroimage. 2001;14(6):1238-1243. doi:10.1006/nimg.2001.0961

9. Mummery CJ, Patterson K, Price CJ, Ashburner J, Frackowiak RS, Hodges JR. A voxel-based morphometry study of semantic dementia: relationship between temporal lobe atrophy and semantic memory. Ann Neurol. 2000;47(1):36-45.

10. Baron JC, Chetelat G, Desgranges B, et al. In vivo mapping of gray matter loss with voxel-based morphometry in mild Alzheimer's disease. Neuroimage. 2001;14(2):298-309. doi:10.1006/nimg.2001.0848
11. Liu H, Wang L, Geng Z, et al. A voxel-based morphometric study of age- and sex-related changes in white matter volume in the normal aging brain. Neuropsychiatr Dis Treat. 2016;12(1):453. doi:10.2147/ NDT.S90674

12. Seo SW, Ahn J, Yoon U, et al. Cortical thinning in vascular mild cognitive impairment and vascular dementia of subcortical type. J Neuroimaging. 2010;20(1):37-45. doi:10.1111/j.1552-6569.2008.00293.x

13. Li C, Du H, Zheng J, Wang J. A voxel-based morphometric analysis of cerebral gray matter in subcortical ischemic vascular dementia patients and normal aged controls. Int J Med Sci. 2011;8(6):482-486.

14. Ai Q, Pu YH, Sy C, Liu LP, Gao PY. Impact of regional white matter lesions on cognitive function in subcortical vascular cognitive impairment. Neurol Res. 2014;36(5):434-443. doi:10.1179/1743132814Y. 0000000354

15. Linortner P, Fazekas F, Schmidt R, et al. White matter hyperintensities alter functional organization of the motor system. Neurobiol Aging. 2012;33(1):197.e1-e9. doi:10.1016/j.neurobiolaging.2010.06.005

16. Katzman R, Zhang MY, Ouang-Ya-Qu, et al. A Chinese version of the Mini-Mental State Examination; impact of illiteracy in a Shanghai dementia survey. J Clin Epidemiol. 1998;41(10):971-978. doi:10.1016/ 0895-4356(88)90034-0

17. Zhang J, Guo Z, Liu X, et al. Abnormal functional connectivity of the posterior cingulate cortex is associated with depressive symptoms in patients with Alzheimer's disease. Neuropsychiatr Dis Treat. 2017; 13(1):2589-2598. doi:10.2147/NDT.S146077

18. Nasreddine ZS, Phillips NA, Bédirian V, et al. The Montreal Cognitive Assessment, MoCA: a brief screening tool for mild cognitive impairment. J Am Geriatr Soc. 2005;53(4):695-699. doi:10.1111/j.15325415.2005.53221.x

19. Lin L, Xue Y, Duan Q, et al. Microstructural white matter abnormalities and cognitive dysfunction in subcortical ischemic vascular disease: an atlas-based diffusion tensor analysis study. J Mol Neurosci. 2015;56(2):363-370. doi:10.1007/s12031-015-0550-5

20. Prins ND, Scheltens P. White matter hyperintensities, cognitive impairment and dementia: an update. Nat Rev Neurol. 2015;11(3):157-165. doi:10.1038/nrneurol.2015.10

21. Launer LJ. Epidemiology of white matter lesions. Top Magn Reson Imaging. 2004;15(6):99-103. doi:10.1097/01.rmr.0000168216. $98338.8 \mathrm{~d}$

22. Vernooij MW, Ikram MA, Vrooman HA, et al. White matter microstructural integrity and cognitive function in a general elderly population. Arch Gen Psychiatry. 2009;66(5):545-553. doi:10.1001/ archgenpsychiatry.2009.5

23. Kuller LH, Longstreth WT Jr, Arnold AM, et al; Cardiovascular Health Study Collaborative Research Group. White matter hyperintensity on cranial magnetic resonance imaging: a predictor of stroke. Stroke. 2004;35(8):1821-1825. doi:10.1161/01.STR.0000132193. 35955.69

24. Sierra C, Coca A. White matter lesions and cognitive impairment as silent cerebral disease in hypertension. Sci World J. 2006;6(1):494-501. doi:10.1100/tsw.2006.99

25. Dong C, Nabizadeh N, Caunca M, et al. Cognitive correlates of white matter lesion load and brain atrophy: the Northern Manhattan Study. Neurology. 2015;85(5):441-449. doi:10.1212/WNL.000000 0000001716

26. Lambert C, Benjamin P, Zeestraten E, Lawrence AJ, Barrick TR, Markus HS. Longitudinal patterns of leukoaraiosis and brain atrophy in symptomatic small vessel disease. Brain. 2016;139(Pt 4):1136-1151. doi:10.1093/brain/aww009

27. Arvanitakis Z, Fleischman DA, Arfanakis K, Leurgans SE, Barnes LL, Bennett DA. Association of white matter hyperintensities and gray matter volume with cognition in older individuals without cognitive impairment. Brain Struct Funct. 2016;221(4):2135-2146. doi:10.1007/ s00429-015-1034-7

28. Raji CA, Lopez OL, Kuller LH, et al. White matter lesions and brain gray matter volume in cognitively normal elders. Neurobiol Aging. 2012;33(4):834.e7-e16. doi:10.1016/j.neurobiolaging.2011.08.010 
29. Muller M, Appelman AP, van der Graaf Y, Vincken KL, Mali WP, Geerlings MI. Brain atrophy and cognition: interaction with cerebrovascular pathology? Neurobiol Aging. 2011;32(5):885-893. doi:10.1016/j. neurobiolaging.2009.05.005

30. Wen W, Sachdev PS, Chen X, Anstey K. Gray matter reduction is correlated with white matter hyperintensity volume: a voxel-based morphometric study in a large epidemiological sample. Neuroimage. 2006; 29(4):1031-1039. doi:10.1016/j.neuroimage.2005.08.057

31. Godin O, Maillard P, Crivello F, et al. Association of white-matter lesions with brain atrophy markers: the three-city Dijon MRI study. Cerebrovasc Dis. 2009;28(2):177-184. doi:10.1159/000226117

32. Mueller SG, Mack WJ, Mungas D, et al. Influences of lobar gray matter and white matter lesion load on cognition and mood. Psychiatry Res. 2010;181(2):90-96. doi:10.1016/j.pscychresns.2009.08.002

33. Kober H, Ochsner KN. Prefrontal-striatal pathway underlies cognitive regulation of craving. Proc Natl Acad Sci U S A. 2010;107(33): 14811-14816. doi:10.1073/pnas.1007779107
34. Wang S, Yuan J, Guo X, et al. Correlation between prefrontal-striatal pathway impairment and cognitive impairment in patients with leukoaraiosis. Medicine (Baltimore). 2017;96(17):e6703. doi:10.1097/MD. 0000000000006703

35. Green MF, Kern RS, Braff DL, Mintz J. Neurocognitive deficits and functional outcome in schizophrenia: are we measuring the "right stuff"? Schizophr Bull. 2000;26(1):119-136.

36. Stern Y, Alexander GE, Prohovnik I, Mayeux R. Inverse relationship between education and parietotemporal perfusion deficit in Alzheimer's disease. Ann Neurol. 1992;32(3):371-375. doi:10.1002/ana.410320311

37. Sandry J, DeLuca J, Chiaravalloti N. Working memory capacity links cognitive reserve with long-term memory in moderate to severe TBI: a translational approach. J Neurol. 2015;262(1):59-64. doi:10.1007/ s00415-014-7523-4

38. Tana C, Tafuri E, Tana M, et al. New insights into the cardiovascular risk of migraine and the role of white matter hyperintensities: is gold all that glitters? J Headache Pain. 2013;14(1):9. doi:10.1186/1129-2377-14-9
Neuropsychiatric Disease and Treatment

\section{Publish your work in this journal}

Neuropsychiatric Disease and Treatment is an international, peerreviewed journal of clinical therapeutics and pharmacology focusing on concise rapid reporting of clinical or pre-clinical studies on a range of neuropsychiatric and neurological disorders. This journal is indexed on PubMed Central, the 'PsycINFO' database and CAS,

\section{Dovepress}

and is the official journal of The International Neuropsychiatric Association (INA). The manuscript management system is completely online and includes a very quick and fair peer-review system, which is all easy to use. Visit http://www.dovepress.com/testimonials.php to read real quotes from published authors.

Submit your manuscript here: http://www.dovepress.com/neuropsychiatric-disease-and-treatment-journal 